\title{
The Role of Trans-Cranial Approach in the Management of Spontaneous CSF Fistula
}

\author{
Waleed Abbass, Mohamed Adel Ghoneim* \\ Department of Neurosurgery, Faculty of Medicine, Cairo University, Cairo, Egypt \\ Email: *madel1981@hotmail.com
}

How to cite this paper: Abbass, W. and Ghoneim, M.A. (2020) The Role of TransCranial Approach in the Management of Spontaneous CSF Fistula. Open Journal of Modern Neurosurgery, 10, 230-236. https://doi.org/10.4236/ojmn.2020.102024

Received: January 5, 2020

Accepted: February 25, 2020

Published: February 28, 2020

Copyright $\odot 2020$ by author(s) and Scientific Research Publishing Inc. This work is licensed under the Creative Commons Attribution International License (CC BY 4.0).

http://creativecommons.org/licenses/by/4.0/

(c) (i) Open Access

\begin{abstract}
Background: Spontaneous descent of the CSF (Cerebrospinal fluid) from the nose is called rhinorrhea. The causes are defects in bones of the base or meningeal tear. The surgical management is still a challenge. The surgical repair is a must to prevent the complication which could be brain abscess, meningitis, or pneumocephalus. The available surgical management is either transnasal, or transcranial. The endonasal route represents an easy access but equally aggressive alternative and of limited access. Objective: We reviewed the clinical outcomes of thirteen cases of spontaneous CSF leakage managed by transcranial intradural approach. Patients and Methods: This is a retrospective study about the records of 13 patients who underwent transcranial intradural approach as a surgical management for spontaneous CSF rhinorrhea in our department in the period between November 2017 and October 2019. Results: CSF leakage stopped after initial surgery in 12 of 13 patients, giving a success rate of $92.3 \%$. The remaining case did not agree for new surgery and the case resolved without surgery for this patient. Postoperative complications included superficial wound infection in one patient, and anosmia in one patient. No other neurological problems were encountered over the ten-months' follow-up period. Conclusion: The transcranial route is the treatment of choice for patients with anterior cranial fossa spontaneous CSF rhinorrhea. A satisfactory surgical outcome depends on exact diagnosis, proper operative approach, and the surgeon's skills and experience.
\end{abstract}

\section{Keywords}

CSF, Trans-Cranial, Intra Dural

\section{Introduction}

Cerebrospinal fluid leakage is due to defects of all the layers that separate the 
subarachnoid space from the upper parts of the respiratory and digestive tracts, namely, duramater, and arachnoid membrane, the mucosa of the nasal cavity or paranasal sinus, cranial base [1] [2].

CSF leakage from the anterior skull base is a common problem that faces most neurosurgeons in their daily practice. One of the most common causes is post traumatic [2] leak, but it is not discussed in our study. Among other common causes is CSF leak that occurs due to either increase in the intracranial pressure due to brain tumors or intracerebral haematomas. Spontaneous rhinorrhea is usually due to a defect in the skull base and this condition may lead to serious complications, such as brain infections or pneumocephalus [1] [2] [3].

The key for successful management and preventing the serious complication is identifying the site of the leak [4]. The surgical transcranial route has been used for more than eighty years since described by Dandy. This strategy allows us to manage any other facial fractures or nerve injury and for this, it has been used so frequent till now [2] [3] [4] [5].

Although the endoscopic technique has a high rate of success and low morbidity, limitations still exist [1] [2] [3] [4]. Patients with CSF leak present with a variety of symptoms, including clear nasal discharge and headache, and may develop complications such as pneumocephalus, meningitis, or brain abscess [6] [7]. Meningitis may occur in $20 \%$ of patients.

With such increase in the duration of leakage without proper management, this will increase the incidence of complication, although it is reported that with no apparent site of leak, meningitis still can occur mainly due to the intermittent leakage [8] [9] [10].

\section{Patients and Methods}

This is a retrospective study the records of 13 patients who underwent transcranial intradural approach as a surgical management for spontaneous CSF rhinorrhea in our department in the period between November 2017 and October 2019 all the patients underwent to full history and neurological examination. The study included all patients with spontaneous leak with failure of medical treatment either denovo or after failed endoscopic repair. The study excluded all the patients with obvious cause of leak such as intra-cranial mass or hydrocephalus (as proven with the neuro imaging) leading to increase in the intra cranial pressure, patients without clear definitive medical treatment and lumbar tap, and any patient with history of trauma. All the patients involved in this research were subjected to high-resolution computed tomography with 1-mm slice thickness and three-dimensional (3D) Reconstruction of the cranial base. Glucose or Beta-2 transferrin fluid were measured as a chemical diagnosis and a proof of spontaneous CSF leakage when the diagnosis is uncertain the other diagnostic tools used in these condition are CT cisternography we may need to use also radionuclide scan and this happened in four cases in this study.

In this study, we followed the same surgical technique in all the patients which 
is bifrontal intradural repair of CSF leakage. The patient is placed in supine posi-tion with the head fixed by Mayfield holder in neutral position. The neck ex-tended with the head elevated above level of heart for adequate venous drainage and to avoid excessive retraction on the frontal lobe. A bicoronal incision behind the hair was made; a vascularized pericranium, flap is prepared. A burr-hole was placed just posterior to the anterior temporal line and midline basal burr-hole over the frontal air sinus and then a bifrontal craniotomy is done flush with the anterior cranial base. The most anterior part of superior sagital sinus is ligated to be cutted. When the frontal air sinus is opened, great attention is taken to remove the whole mucosa and is packed with fat and gelfoam soaked with betadine. The sphenoid ridge is drilled. The dura is opened in a linear fashion flushed with the base. Care must be taken to drain CSF to avoid excessive retraction of both frontal lobes; we begin by the right frontal lobe and after inspection of right cranial fossa base; the same steps are done on the left side. If the tension is high CSF drainage is done by opening of optico-carotid cistern or proximal opening of sylvian fissure. Identification of the defect is done, and then we start the repair of the base using three layers. $\$ 1$ st layer: By autolysis fat fixed to the base by histoacryl $\$ 2$ nd layer: By fascia lata we sutured the upper edge of the fascia lata to the anterior edge of the dura and the lower edge is put onlay on the anterior cranial base and fixed by histoacryl $₫$ 3rd layer: By vascularized pericranium flap, where the lower edge is put onlay on the base and fixed by histoacryl. Then the vascularized pericranium flap is sutured to the posterior edge of the dura the bone flap is restored in position and we use daily lumbar draindage by doing lumbar puncture and draining about thirty $\mathrm{ml} /$ every day for only five days with proper anti biotic coverage.

\section{Results}

This is a retrospective study of 13 patients presented to our departments suffering from spontaneous anterior cranial fossa leakage. The study included 9 males and 4 females their age ranged from 18 years to 42 years with the mean of 30 years. Eight cases were referred from the ENT departments after failure of primary repair via the endo-nasal endoscopic approach (Figure 1 and Figure 2), the remaining of the cases, three of them had big basal defect, and two cases there was no noticeable defect (radiologically) in the base. In our series, the most common presentation was a watery like nasal secretion with salty taste headache (in three patients) was the second common presenting symptom it may be caused by pneumocephalus.

The detected defects during surgery in the eleven cases were in the ethmoid-cribriform plate, others 2 cases no defect could be found and repair was done as usual (Table 1).

Surgery is considered with good results (if no nasal leakage or intra cranial infection occurred in the follow up period). Post operative 12 cases went with no leak through all the follow up duration one case developed intermittent leak after 2 weeks reoperation was proposed but the patient refused, and with 

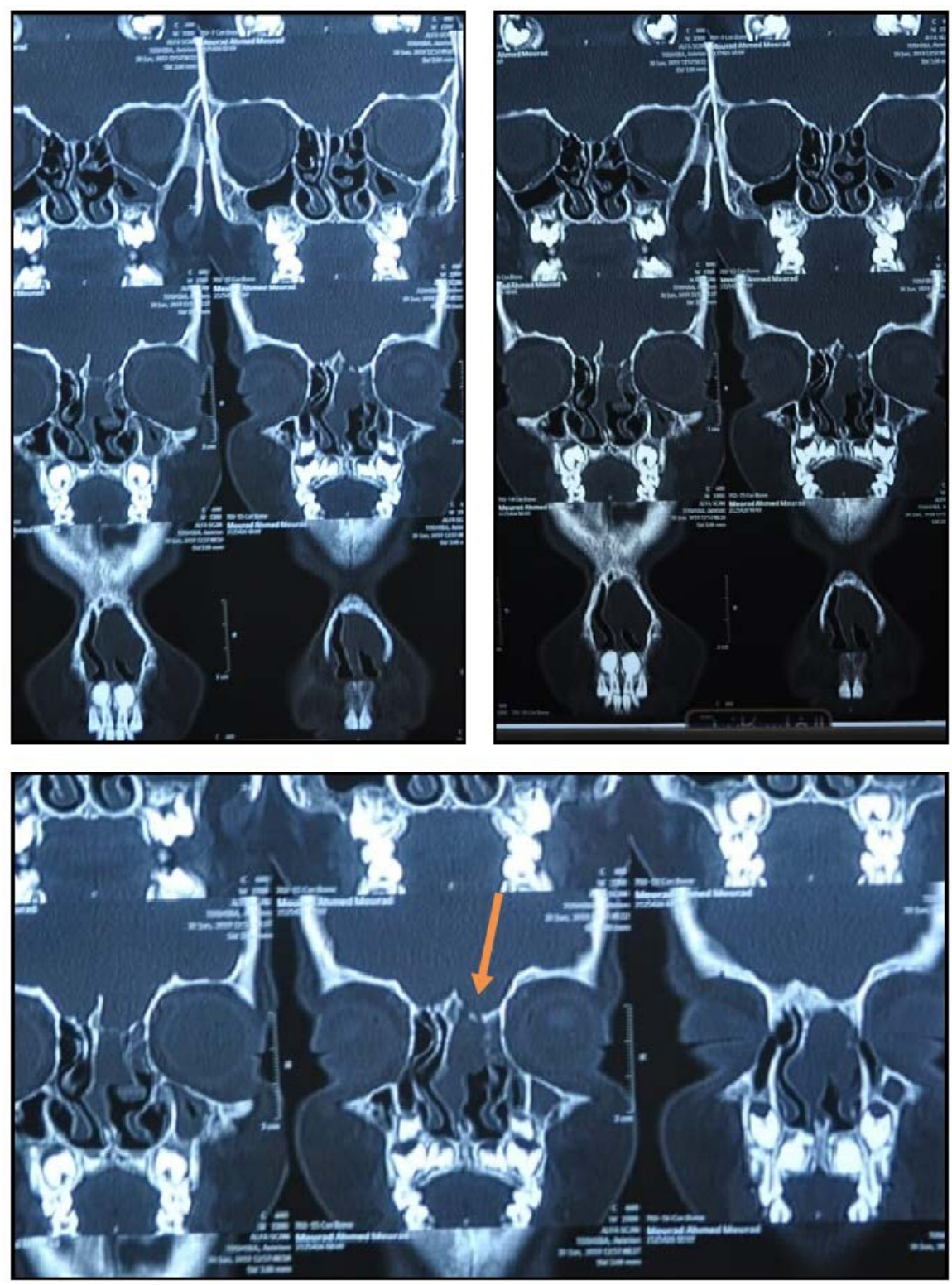

Figure 1. Pre operative images CT coronal cuts in female patient 33 years with history of CSF leakage from left nostril and failed endoscopic repair (arrow on the defect in the left ethmoid-cribriform plate).

medical treatment and infrequent lumbar puncture the condition resolved, so our management was successful in $92.3 \%$, we also did not encountered any neurological problems due to brain oedema or haematoma.

Others complications one patient had superficial wound infection and resolved by frequent dressing, one patient had anosmia before the operation intraoperative the nerve was injured but postoperative passed unnoticed so it was not listed as a neurological complication. Three patients had in follow up immediate after the operation pneumocephalus, which were present preoperative and were managed by mask of oxygen and analgesics and it was relieved (Table 2). 


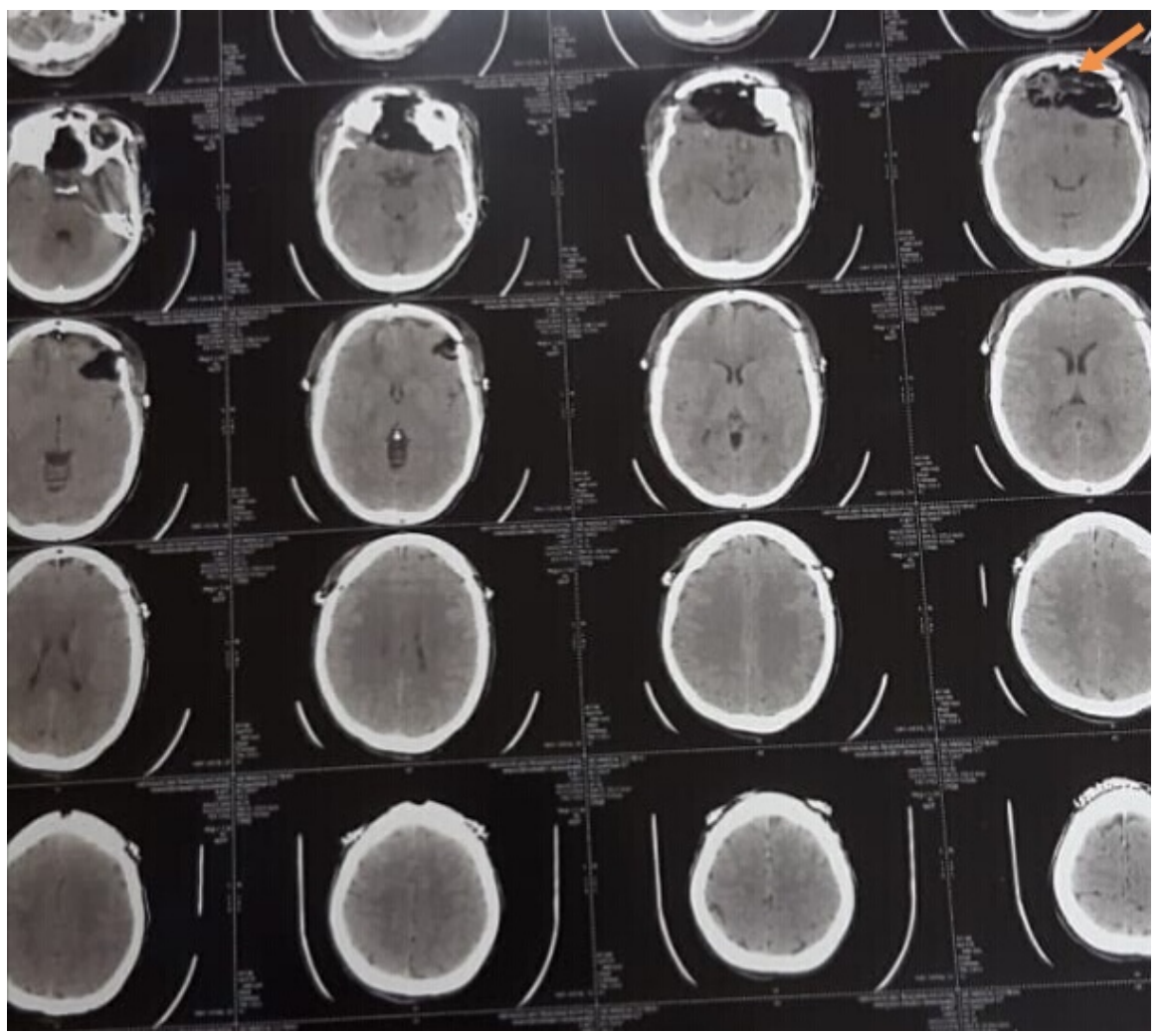

Figure 2. Post operative follow up CT Brain (arrow on the 3 layers of repair which were fat, fascia lata, and vascularized pericranium).

Table 1. Demographic, clinical characteristics, and defects during surgery of the participants.

\begin{tabular}{cl}
\hline Gender & 9 males \\
& 4 females \\
& 18 to 42 years \\
& Mean age 30 years \\
& 8 cases failed endoscopic approach \\
& 3 cases big basal defect \\
Patients & 2 no noticeable defect (radiologically) \\
& Watery like nasal secretion with salty taste in all patients \\
Common presentation & Headache 9 patients \\
& 11 cases in the ethmoid-cribriform plate. \\
Defects during surgery & 2 cases defect couldn't found \\
\hline
\end{tabular}

Table 2. Complications.

\begin{tabular}{ll}
\hline & 1 case developed leak after 2 weeks \\
& 1 patient had superficial wound infection \\
& 1 patient had anosmia \\
& 3 patient had pneumocephalus \\
\hline
\end{tabular}

\section{Discussion}

CSF fistulae, encephaloceles, and meningoceles occur either spontaneously or 
due to damage to the skull base, the management of these lesions is indicated for the prevention of complications such as pneumocephalus meningitis, and brain abscess [2]-[7].

Although traditionally, operative approaches for management of this condition have included transcranial and endonasal microscopic methods, which still have limited access although the good results recorded. the surgical trans cranial approach is superior to the trans nasal route as we can explore the base of the skull either intra dural or extra dural inspection if there is associated fractures they could be managed as well if there is involvement of any of the basal structures beside the main purpose of the operation to close the communication between the intra cranial cavity and the extra cranial compartment [3]-[9].

The material could be used to seal the big defects in the base are variable could be fascia lata or pericranium temporalis muscle fascia could be used some surgeons may prefer fibrin glue we preferred viable pericranial flaps in our study because they are integrated easily and resistant to infection and provide a good watertight sealant and it is the most recommended in the literature [10]. We favour bilateral craniotomy even if radiological studies show unilateral dural defects, because dural tears on the contra lateral side are not rare, and both olfactory bulbs may be injured In our study we used antibiotics for five days in the post operative periods although there is great controversial about their role and one study recommended not to use them as not obvious role in preventing meningitis during the transcranial approach a great care is giving in cranialization of the frontal air sinus if it was intruded during the bony work of the bone flap removal of the mucosa to prevent the occurrence of mucocele and the nasofrontal duct should be obliterated to prevent any communication in the literature the decrease in the CSF tension after the operation is highly recommended by many surgeons specially if we are dealing with big basal defects in our review repeated lumbar puncture was the mainstay some recommend continues lumbar drain but we preferred the lumbar puncture it is less dangerous considering the complications such as headache vomiting or even brain herniation but it is more traumatizing [3]-[9].

\section{Conclusion}

With the rising use of endoscopic repair of spontaneous CSF leak trans-cranial repair is still effective and with no limited access easily done and with higher successful rate.

\section{Conflicts of Interest}

The authors declare no conflicts of interest regarding the publication of this paper.

\section{References}

[1] Lindstrom, D.R., Toohill, R.J., Loehrl, T.A. and Smith, T.L. (2004) Management of 
Cerebrospinal Fluid Rhinorrhea: The Medical College of Wisconsin Experience. Laryngoscope, 114, 969-974. https://doi.org/10.1097/00005537-200406000-00003

[2] Nyquist, G.G., Anand, V.K., Mehra, S., Kacker, A. and Schwartz, T.H. (2010) Endoscopic Endonasal Repair of Anterior Skull Base Non-Traumatic Cerebrospinal Fluid Leaks, Meningoceles, and Encephaloceles. Journal of Neurosurgery, 113, 961-966. https://doi.org/10.3171/2009.10.JNS08986

[3] Tabaee, A., Kassenoff, T.L., Kacker, A. and Anand, V.K. (2005) The Efficacy of Computer Assisted Surgery in the Endoscopic Management of Cerebrospinal Fluid Rhinorrhea. Otolaryngology-Head and Neck Surgery, 133, 936-943. https://doi.org/10.1016/j.otohns.2005.07.028

[4] Arai, A., Mizukawa, K., Nishihara, M., Fujita, A., Hosoda, K. and Kohmura, E. (2010) Spontaneous Cerebrospinal Fluid Rhinorrhea Associated with a Far Lateral Temporal Encephalocele-Case Report. Neurologia Medico-Chirurgica (Tokyo), 50, 243-245. https://doi.org/10.2176/nmc.50.243

[5] Bell, R.B., Dierks, E.J., Homer, L. and Potter, B.E. (2004) Management of Cerebrospinal Fluid Leak Associated with Craniomaxillofacial Trauma. Journal of Oral and Maxillofacial Surgery, 62, 676-684. https://doi.org/10.1016/j.joms.2003.08.032

[6] Bibas, A.G., Skia, B. and Hickey, S.A. (2000) Transnasal Endoscopic Repair of Cerebrospinal Fluid Rhinorrhea. British Journal of Neurosurgery, 14, 49-52. https://doi.org/10.1080/02688690042924

[7] Eftekhar, B., Ghodsi, M., Nejat, F., Ketabchi, E. and Esmaeeli, B. (2004) Prophylactic Administration of Ceftriaxone for the Prevention of Meningitis after Traumatic Pneumocephalus: Results of a Clinical Trial. Journal of Neurosurgery, 101, 757-761. https://doi.org/10.3171/jns.2004.101.5.0757

[8] Katzen, J.T., Jarrahy, R., Eby, J.B., Mathiasen, R.A., Margulies, D.R. and Shahinian, H.K. (2003) Craniofacial and Skull Base Trauma. The Journal of Trauma, 54, 1026-1034. https://doi.org/10.1097/01.TA.0000066180.14666.8B

[9] Locatelli, D., Rampa, F., Acchiardi, I., Bignami, M., De Bernardi, F. and Castelnuovo, P. (2006) Endoscopic Endonasal Approaches for Repair of Cerebrospinal Fluid Leaks: Nine-Year Experience. Neurosurger, 58, 246-257. https://doi.org/10.1227/01.NEU.0000193924.65297.3F

[10] Rocchi, G., Caroli, E., Belli, E., Salvati, M., Cimatti, M. and Delfini, R. (2005) Severe Craniofacial Fractures with Frontobasal Involvement and Cerebrospinal Fluid Fistula: Indications for Surgical Repair. Surgical Neurology, 63, 559-563.

https://doi.org/10.1016/j.surneu.2004.07.047 